\title{
Metabolic engineering of Saccharomyces cerevisiae ethanol strains PE-2 and CAT-1 for efficient lignocellulosic fermentation
}

\author{
Aloia Romaní $^{\mathrm{a}}$, Filipa Pereira ${ }^{\mathrm{b}}$, Björn Johansson ${ }^{\mathrm{b}, 1}$, Lucília Domingues ${ }^{\mathrm{a}, *, 1}$ \\ ${ }^{a}$ CEB - Centre of Biological Engineering, University of Minho, Campus de Gualtar, Braga 4710-057, Portugal \\ ${ }^{\mathrm{b}}$ CBMA - Center of Molecular and Environmental Biology, University of Minho, Campus de Gualtar, Braga 4710-057, Portugal
}

\section{H I G H L I G H T S}

- PE-2 and CAT-1 S. cerevisiae strains were engineered for xylose fermentation.

- Engineered PE-2 (MEC1121) strain consumed xylose and glucose simultaneously.

- MEC1121 strain was able to efficiently ferment xylose in the presence of inhibitors.

- Deletion of the aldose reductase GRE3 (MEC1133 strain) lowered xylitol production.

- High ethanol yield was achieved by MEC1133 in SSCF of corn cob hydrolysate.

\section{A R T I C L E I N F O}

\section{Article history:}

Received 29 October 2014

Received in revised form 5 December 2014

Accepted 7 December 2014

Available online 12 December 2014

\section{Keywords:}

Second generation bio-ethanol

Xylose fermentation

GRE3 aldose reductase

Industrial Saccharomyces cerevisiae

Simultaneous saccharification and co-

fermentation (SSCF)

\begin{abstract}
A B S T R A C T
In this work, Saccharomyces cerevisiae strains PE-2 and CAT-1, commonly used in the Brazilian fuel ethanol industry, were engineered for xylose fermentation, where the first fermented xylose faster than the latter, but also produced considerable amounts of xylitol. An engineered PE-2 strain (MEC1121) efficiently consumed xylose in presence of inhibitors both in synthetic and corn-cob hydrolysates. Interestingly, the S. cerevisiae MEC1121 consumed xylose and glucose simultaneously, while a CEN.PK based strain consumed glucose and xylose sequentially. Deletion of the aldose reductase GRE3 lowered xylitol production to undetectable levels and increased xylose consumption rate which led to higher final ethanol concentrations. Fermentation of corn-cob hydrolysate using this strain, MEC1133, resulted in an ethanol yield of $0.47 \mathrm{~g} / \mathrm{g}$ of total sugars which is $92 \%$ of the theoretical yield.
\end{abstract}

(c) 2014 Elsevier Ltd. All rights reserved.

\section{Introduction}

Climate changes and a possible future shortage of fossil petroleum have motivated a continuous development of biomass derived biofuels for the last twenty years. In fact, the use of $10 \%$ renewable fuels for transport is part of targets of climate and energy policy of EU by 2020. Nowadays, the bioethanol from food crops is the main worldwide biofuel produced and commercialized (van Eylen et al., 2011). However, competition with agriculture for limited farmland for this feedstock requires the use of other raw materials (Tomás-Pejó et al., 2014). In this context, renewable raw materials, such as lignocellulosic materials (LCM), represent

\footnotetext{
* Corresponding author.

E-mail addresses: aloia@ceb.uminho.pt (A. Romaní), filipaabpereira@gmail.com (F. Pereira), bjorn_johansson@bio.uminho.pt (B. Johansson), luciliad@deb.uminho. pt (L. Domingues).

${ }^{1}$ Both authors contributed equally to the work.
}

an available feedstock in sufficient amounts to partially replace fossil fuels.

The hydrolysis of LCM breaks down the lignocellulosic structure (formed by cellulose, hemicellulose and lignin) and the solubilization of these polysaccharides into fermentable sugars. The pretreatment or processing of LCM allows the improvement of enzymatic access to cellulose and the solubilization of hemicellulose into oligosaccharides and monosaccharides (Romaní et al., 2014). Nevertheless, the extraction of sugars by acidic thermochemical pretreatments implies the release of other undesirable compounds (as weak acids, furans and phenolic compounds) that are considered inhibitors of ethanol fermentation (Pereira et al., 2014).

The hemicellulose fraction of LCM is constituted partly of xylan and, especially in hardwood and agro-industrial wastes, arabinoxylan, which are polymers of the pentose sugars D-xylose and Larabinose. These pentose sugars can constitute up to $40 \%$ of total sugars in LCM hydrolysate (Tomás-Pejó et al., 2014). D-xylose and L-arabinose are metabolized by many naturally occurring 
microorganisms, but not by the preferred industrial ethanol production organism Saccharomyces cerevisiae. Cost-effective production of ethanol from LCM hydrolysates requires efficient and rapid fermentation of all sugars present in the lignocellulosic biomass (Kim et al., 2013). Xylose fermentation would decrease the cost of ethanol production by $25 \%$ (Hinman et al., 1989). S. cerevisiae was first engineered for D-xylose fermentation by the expression of the genes SsXYL1 and SsXYL2 from Scheffersomyces stipitis encoding D-Xylose reductase (XR) and xylitol dehydrogenase (XDH), respectively (Kötter et al., 1990). XR catalyze the reduction of D-xylose to xylitol with NADPH or NADH as cofactor, while XDH oxidizes xylitol to D-xylulose using NAD+ in an overall redox neutral isomerization. Expression of SsXYL1 and SsXYL2 alone resulted in aerobic growth on D-xylose (Kötter et al., 1990), but very little ethanol production and production of considerable amounts of xylitol (Kim et al., 2013). The xylitol produced has two main sources, a cofactor imbalance between the XR and XDH enzymes (Kim et al., 2013) and the unspecific aldose reductase GRE3 which reduce D-xylose to xylitol using NADPH as cofactor (Träff et al., 2001). A number of strategies have been devised to lower xylitol byproduct formation. Xylose isomerase can carry out the isomerization of xylose to xylulose without xylose intermediate, reducing xylitol formation (Kuyper et al., 2003). Alternatively, D-xylose reductase from $S$. stipitis was engineered by random mutagenesis followed by selection in anaerobic batch culture and a mutated XR (N272D) was able to diminish the cofactor imbalance caused in the first two steps of xylose metabolism, which improved the ethanol yield and productivity. This mutant D-xylose reductase enzyme has higher specificity for NADH than NADPH (Runquist et al., 2010). Finally, GRE3 deletion removes the principal native route of xylitol production (Träff et al., 2001).

Moreover, fermentation performance is also dependent on intrinsic strain characteristics that are not easy to pinpoint. Genetically tractable laboratory strains normally do not perform well in industrial fermentations. One of the first examples of industrial $S$. cerevisiae strains modified for xylose fermentation were the TMB3006 (Hahn-Hägerdal and Pamment, 2004), based on a strain isolated from a fermentation line of a sulfite pulp mill (Lindén et al., 1992) and TMB3400 (Wahlbom et al., 2003), based on the wine yeast hybrid USM21 (van der Westhuizen and Pretorius, 1992). The TMB3400 consumed D-xylose faster than comparable laboratory strain (Sonderegger et al., 2004). Another interesting source of potentially robust strains is the current Brazilian ethanol industry. S. cerevisiae PE-2 and CAT-1 were isolated from Brazilian industrial ethanol plants in 1994 and 1998, respectively (Basso et al., 2008). The PE-2 was reported in 2009 to be used by roughly a third of Brazilian distilleries, generating about $10 \%(\mathrm{v} / \mathrm{v})$ fermented ethanol in the world (Argueso et al., 2009). In addition, these strains have been shown to have unique physiological characteristics (Pereira et al., 2011) conferring general robustness. Interestingly, we recently found that these strains efficiently ferment lignocellulosic hydrolysates (Pereira et al., 2014). During the course of fermentation, PE-2 was able to reduce hydroxymethylfurfural and furfural content of a hydrolysate medium from autohydrolysis of Eucalyptus globulus further than any of the other strains tested (Pereira et al., 2014) but xylose was not metabolized.

Taking advantage of the robust background of the Brazilian $S$. cerevisiae strains, the aim of this work was to construct efficient $S$. cerevisiae strains engineered for D-xylose fermentation. Finally, selected engineered strains were evaluated for ethanol production using corn cob hydrolysate in simultaneous saccharification and co-fermentation (SSCF).

\section{Methods}

\subsection{Strains, media and cell cultivation for genetic constructions}

Escherichia coli XL1-Blue (Stratagene) cultivated was used to propagate plasmids on solid Lysogeny Broth (LB) medium supplemented with $100 \mathrm{mg} / \mathrm{L}$ ampicillin. Liquid cultures were incubated at $37{ }^{\circ} \mathrm{C}$ on a rotational shaker at $200 \mathrm{rpm}$. Yeast DNA transformation was carried out using a high efficiency transformation protocol by lithium acetate/single-stranded carrier DNA/polyethylene glycol method (Gietz and Schiestl, 2007). E. coli strains were transformed with the simple and efficient method (SEM) (Inoue et al., 1990). Yeast and E. coli strains used in this work are listed in

Table 1

Strains and plasmids used in this work.

\begin{tabular}{|c|c|c|}
\hline & Relevant genotype & References \\
\hline \multicolumn{3}{|l|}{ Yeast strains } \\
\hline CEN.PK 113 5D & MATa, ura3-52 & van Dijken et al. (2000) \\
\hline PE-2 & Diploid; Isolated from ethanol plants in 1994 & Basso et al. (2008) \\
\hline CAT-1 & Diploid; Isolated from ethanol plants in 1998 & Basso et al. (2008) \\
\hline MEC1134 & CEN.PK 113 5D, pMEC149 & This work \\
\hline MEC1121 & PE-2, pMEC149 & This work \\
\hline MEC1122 & CAT-1, pMEC149 & This work \\
\hline Y01932 ( $\Delta$ gre3) & BY4741; Mat a; his $3 \Delta 1$; leu2 $\Delta 0$; met15 $\Delta 0$; ura3 $\Delta 0$; YHR104w::kanMX4 & EUROSCARF \\
\hline MEC1131 & Y01932, YHR104w::natMX4 & This work \\
\hline MEC1132 & PE-2, gre3::natMX4 / GRE3, pMEC149 & This work \\
\hline MEC1133 & PE-2, gre3::natMX4 / gre3::kanMX4, pMEC149 & This work \\
\hline \multicolumn{3}{|l|}{ E. coli strains } \\
\hline XL1BLUE & & Stratagene \\
\hline Plasmids & Property & \\
\hline pAG32 & HphMX4, amp & Goldstein and McCusker (1999) \\
\hline pAG25 & NatMX4, amp & Goldstein and McCusker (1999) \\
\hline pMEC1035 & PYPK0-TEF1tp-XR(N272D)-TDH3tp-XYL2-PGI1tp -XKS1- FBA1tp-TAL1-PDC1tp, URA3 & Pereira et al. (submitted) \\
\hline pMEC1049 & PYPK4-TEF1tp-XR(N272D)-TDH3tp-XYL2-PGI1tp-XKS1-FBA1tp-TAL1-PDC1tp, HphMX4 & This work \\
\hline Primers & Sequence & \\
\hline MX4_fwd & AAAATCTTGCTAGGATACAGTTCTC & \\
\hline MX4_rev & CGACTCACTATAGGGAGACC & \\
\hline A_gre3 & CTATACTTGTTTCCCAATTGTTGCT & \\
\hline D_gre3 & ATTTTAGTATCACTCACTCATCGCC & \\
\hline pYPK0_hygfwd & ctcacgttaagggattttggtcatgagCACATACGATTTAGGTGACACTATAGAAC & \\
\hline pYPK0_hygrev & catctttgacagcttatcatcgataagctCGACTCACTATAGGGAGACC & \\
\hline
\end{tabular}


Table 1. Yeast cells were cultivated in complex YP medium $(20 \mathrm{~g} / \mathrm{L}$ peptone and $10 \mathrm{~g} / \mathrm{L}$ yeast extract) with varying carbon sources (glucose and/or D-xylose) at different concentrations. Solid medium was made by adding $20 \mathrm{~g} / \mathrm{L}$ of technical agar. Media were supplemented with $300 \mathrm{mg} / \mathrm{L}$ of geneticin (G418) or hygromycin or $200 \mathrm{mg} / \mathrm{L}$ of nourseothricin as required. D-xylose metabolizing yeast strains were maintained on YPX plates $(20 \mathrm{~g} / \mathrm{L}$ of xylose) at $4{ }^{\circ} \mathrm{C}$ and re-streaked regularly.

\subsection{Molecular biological methods}

The sequences of PCR primers used are listed in Table 1. Taq DNA Polymerase purchased from Thermo Fisher Scientific Inc. was used for diagnostic PCR while PCR amplifications for construction were performed using Phusion ${ }^{\circledR}$ DNA polymerase obtained from New England BioLabs ${ }^{\circledR}$ Inc. Primer concentration was $1 \mu \mathrm{M}$ of each primer in all PCR reactions. Plasmids propagated in E. coli were purified using the GenElute ${ }^{\mathrm{TM}}$ Plasmid Miniprep kit (SigmaAldrich ${ }^{\circledR}$ Co., LLC). Plasmids in S. cerevisiae were extracted using the same kit but with an additional initial step. Briefly, yeast cells were resuspended in $200 \mu \mathrm{L}$ of solution A, $9 \mathrm{U}$ lyticase (SigmaAldrich ${ }^{\circledR}$ Co., LLC) and $200 \mu \mathrm{L}$ of glass beads $0.4-0.6 \mu \mathrm{m}$ (SigmaAldrich ${ }^{\circledR}$ Co., LLC), incubated at $37^{\circ} \mathrm{C}$ for $30 \mathrm{~min}$. The mixture was subsequently vortexed for $3 \mathrm{~min}$. at room temperature to break the cells. The remaining steps were performed according to the provided protocol. Yeast chromosomal DNA was extracted using the Preparation of Yeast DNA protocol (Hoffman, 2001).

\subsection{Construction of xylose pathway pMEC1049}

In this work a novel metabolic pathway assembly tool called the Yeast Pathway Kit (YPK) was used to construct new D-xylose fermenting $S$. cerevisiae strains (Pereira et al., submitted). This pathway assembly tool is based on hierarchical homologous recombination of fragments cloned in an E. coli positive selection vector. Using YPK the xylose utilizing pathway was assembled in a multi-copy at once into $S$. cerevisiae CEN.PK 113-5D strain, using $U R A 3$ gene as a selection marker. The engineered pathway contains the SsXYL1 (N272D; mutated (Runquist et al., 2010)), SsXYL2, ScXKS1 and ScTAL1 genes, under different $S$. cerevisiae promoters TEF1, TDH3, PGI1 and FBA1, respectively. The obtained plasmid was named pMEC1035 (Pereira et al., submitted). The hphMX4 hygromycin resistance cassette was amplified from plasmid pAG32 (Goldstein and McCusker, 1999) with primers pYPKO_hygfwd and pYPKO_hygrev. The PCR product was transformed together with plasmid pMEC1035 (Pereira et al., submitted) into S. cerevisiae CEN.PK 113-5D (van Dijken et al., 2000). Transformants were plated on selective hygromycin medium. The vector pMEC1049 resulting from homologous recombination has the URA3 selection marker replaced by the hphMX4 hygromycin resistance gene (Goldstein and McCusker, 1999). The S. cerevisiae CEN.PK 113-5D carrying the pMEC1049 plasmid was given the name MEC1134. The pMEC1049 plasmid was extracted from yeast and the crude plasmid DNA preparation was used as PCR template for diagnostic PCR and to transform E. coli. Pure pMEC1049 was isolated from $E$. coli and used to transform $S$. cerevisiae strains PE-2 and CAT-1 and selected transformants were designated MEC1121 and MEC1122, respectively (Table 1).

\section{4. gre3 disruption in PE-2}

The nourseothricin resistance cassette natMX4 was amplified from pAG25 (Goldstein and McCusker, 1999) with primers MX4_fwd and MX4_rev specific for the Ashbya gossypii TEF1 promoter and terminator, respectively. The PCR product was used to transform the S. cerevisiae Y01932 gre3::kanMX4 strain obtained

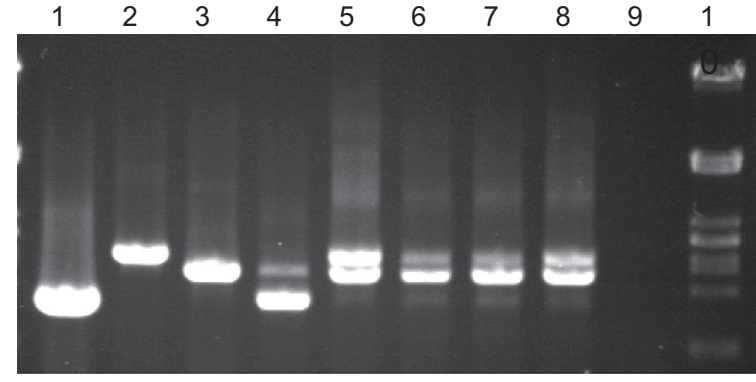

Fig. 1. Agarose gel electrophoresis of $P C R$ products obtained from the amplification of the GRE3 locus using the primers A-gre3 and D-gre3 and chromosomal DNA from different S. cerevisiae strains as template. Lane-1: PE-2(GRE3/GRE3), Lane-2: Y01932 (gre3::kanMX4), Lane-3: Y01932N (gre3::natMX4), Lane-4: MEC1132 (gre3::natMX4/GRE3), Lanes 5-8: four clones of MEC1133 (gre3::natMX4/ gre3::kanMX4), Lane-9: no template, Lane-10: Bacteriophage Lambda DNA digested with PstI.

from the EUROSCARF strain collection. Transformants were selected for nourseothricin resistance and G418 sensitivity. The substitution of the kanMX4 marker for the natMX4 was confirmed by colony PCR. One clone was selected and named MEC1131. Chromosomal DNA from laboratory strains Y01932 and MEC1131 was extracted and used as template to PCR amplify the two deletion cassettes using primers A_gre3 and D_gre3 flanking the gre3::kanMX4 locus in Y01932 and the gre3::natMX4 in MEC1131. The MEC1121 was transformed with the PCR product of natMX4 deletion cassette and plated on YPD medium containing hygromycin and nourseothricin. Transformants were selected and gene deletion as well as a remaining GRE3 locus was confirmed by diagnostic PCR using primers A_gre3 and D_gre3. One clone was selected and named MEC1132. MEC1132 was then transformed with the deletion cassette gre3::kanMX4 to disrupt the remaining GRE3 allele. Transformants were selected on YPD medium containing hygromycin, nourseothricin and geneticin. Diagnostic PCR with primers A_gre3 and D_gre3 confirmed the presence of gre3::kanMX4 and gre3::natMX4 as well as the absence of the GRE3 locus (Fig. 1). The double gre3 mutant containing the xylose pathway was named MEC1133.

\subsection{Fermentation media}

The yeast strains used for inoculum were conserved in YPX plates $(20 \mathrm{~g} / \mathrm{L}$ of xylose, $20 \mathrm{~g} / \mathrm{L}$ of technical agar, $5 \mathrm{~g} / \mathrm{L}$ of yeast extract and $10 \mathrm{~g} / \mathrm{L}$ of peptone) at $4{ }^{\circ} \mathrm{C}$ and plated routinely. The cells for inoculation were grown in $250 \mathrm{~mL}$ Erlenmeyer flasks filled with $100 \mathrm{~mL}$ media containing $30 \mathrm{~g} / \mathrm{L}$ of xylose, $20 \mathrm{~g} / \mathrm{L}$ of peptone and $10 \mathrm{~g} / \mathrm{L}$ of yeast extract in an orbital shaker $(200 \mathrm{rpm})$ at $30^{\circ} \mathrm{C}$ for $24 \mathrm{~h}$. Biomass was collected by centrifugation (for $10 \mathrm{~min}$, $9000 \mathrm{rpm}$ and $4{ }^{\circ} \mathrm{C}$ ), suspended in $0.9 \% \mathrm{NaCl}$ solution to achieve a concentration of $200 \mathrm{mg}$ of fresh yeast $/ \mathrm{mL}$. The fermentation experiments were inoculated with $5 \mathrm{mg}$ cells $/ \mathrm{mL}$ and carried out in $100 \mathrm{~mL}$ Erlenmeyer flasks (working volume $40 \mathrm{~mL}$ ) at $30^{\circ} \mathrm{C}$ in an orbital shaker $(150 \mathrm{rpm})$ under oxygen-limited and anaerobic conditions and $\mathrm{pH}=5$. The difference between these two conditions is that under oxygen-limited conditions the oxygen concentration is $9 \mathrm{mg} / \mathrm{L}$ instead of zero. This amount of oxygen is rapidly consumed by the yeast decreasing to zero in a very short time. Biomass concentration in the media was measured by dry cell weight in the end of fermentation. The experiments of this study were performed in triplicate which allowed the standard deviation calculation. Several synthetic media were used for the evaluation of selected strains, as it is described below:

\subsubsection{Xylose medium}

The media used was composed by YP medium containing $45 \mathrm{~g} / \mathrm{L}$ of xylose. In order to maintain anaerobic conditions, flasks were 
closed with perforated rubber stoppers enclosing glycerol-locks, to allow $\mathrm{CO}_{2}$ exhaustion and avoid the entrance of air. Alternatively, flasks were closed with cotton stopper. Under this condition, defined as oxygen-limited, the culture has contact with air but is under oxygen limitation as in industrial conditions.

\subsubsection{Xylose and glucose medium}

The fermentations were carried out in oxygen-limited conditions using a YP medium containing different concentrations of xylose and glucose: (i) xylose and glucose in equal amounts ( $17 \mathrm{~g} / \mathrm{L}$ of xylose and $17 \mathrm{~g} / \mathrm{L}$ of glucose, Fig. 2a), (ii) more glucose than xylose (38 g/L of glucose and $27 \mathrm{~g} / \mathrm{L}$ of xylose): Fig. 2b), (iii) more xylose than glucose ( $27 \mathrm{~g} / \mathrm{L}$ of xylose and $17 \mathrm{~g} / \mathrm{L}$ of glucose): Fig. 2c and d).

\subsubsection{Synthetic hydrolysate medium}

For the evaluation of yeast fermentation performance in presence of inhibitor compounds, a synthetic hydrolysate was prepared. The synthetic hydrolysate was composed by $30 \mathrm{~g} / \mathrm{L}$ of xylose, $1.9 \mathrm{~g} / \mathrm{L}$ of glucose, $2.3 \mathrm{~g} / \mathrm{L}$ of acetic acid, $1.2 \mathrm{~g} / \mathrm{L}$ of furfural and $0.1 \mathrm{~g} / \mathrm{L}$ of hydroxymethylfurfural (HMF). This composition was based on data obtained from corn cob hydrolysate (Rivas et al., 2002). This medium was supplemented with $30 \mathrm{~g} / \mathrm{L}$ of corn steep liquor (CSL).

\subsubsection{Mineral medium}

In order to compare the results of this work with published data in the literature, selected strains were employed in fermentations carried out in Erlenmeyer flasks filled with $40 \mathrm{~mL}$ of mineral
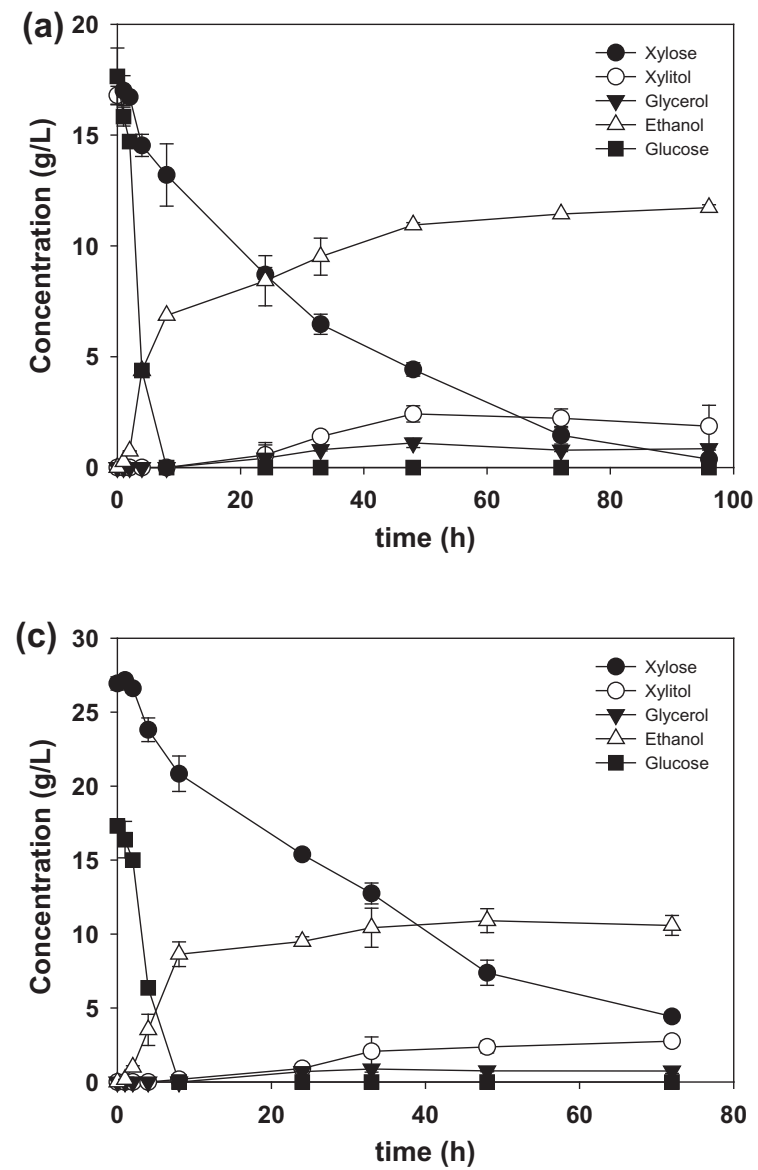

medium (Verduyn et al., 1992), $17 \mathrm{~g} / \mathrm{L}$ of glucose and $40 \mathrm{~g} / \mathrm{L}$ of xylose (Fig. 3a and b).

2.6. Pretreatment corn cob: autohydrolysis and sulfuric acid treatment of autohydrolysate

Corn cob was collected locally, milled and stored until used. The corn cob was submitted to hydrothermal treatment (autohydrolysis) at liquid to solid ratio $8 \mathrm{~kg}$ of water $/ 1 \mathrm{~kg}$ of raw material in a pressurized reactor to reach $202{ }^{\circ} \mathrm{C}$, as described previously (Rivas et al., 2002). The liquid and solid fraction obtained after treatment were separated by filtration and the liquid phase (autohydrolysate) was submitted to sulfuric acid treatment with $0.5 \%$ $\mathrm{H}_{2} \mathrm{SO}_{4}$ for $165 \mathrm{~min}$ at $121^{\circ} \mathrm{C}$ (Rivas et al., 2002). The resulting monomeric sugar solution was neutralized with $\mathrm{CaCO}_{3}$ to a final $\mathrm{pH}$ of 5 and the $\mathrm{CaSO}_{4}$ precipitate was removed by filtration. The monomeric sugar solution was sterilized by filtration $(0.2 \mu \mathrm{m})$. The autohydrolysate and monomeric sugar solution were analyzed for chemical composition by HPLC. The solid fraction resulting from autohydrolysis was analyzed for chemical composition following the procedure described before (Romaní et al., 2014). The chemical composition of the hydrolysate is presented in Fig. 4. All of data presented in Fig. 4 were carried out in triplicate.

\subsection{Simultaneous saccharification and Co-fermentation assays of pretreated corn cob}

The SSCF assays were carried out in $100 \mathrm{~mL}$ Erlenmeyer flasks at $30{ }^{\circ} \mathrm{C}$ with an orbital shaker (150 rpm) using the solid fraction from
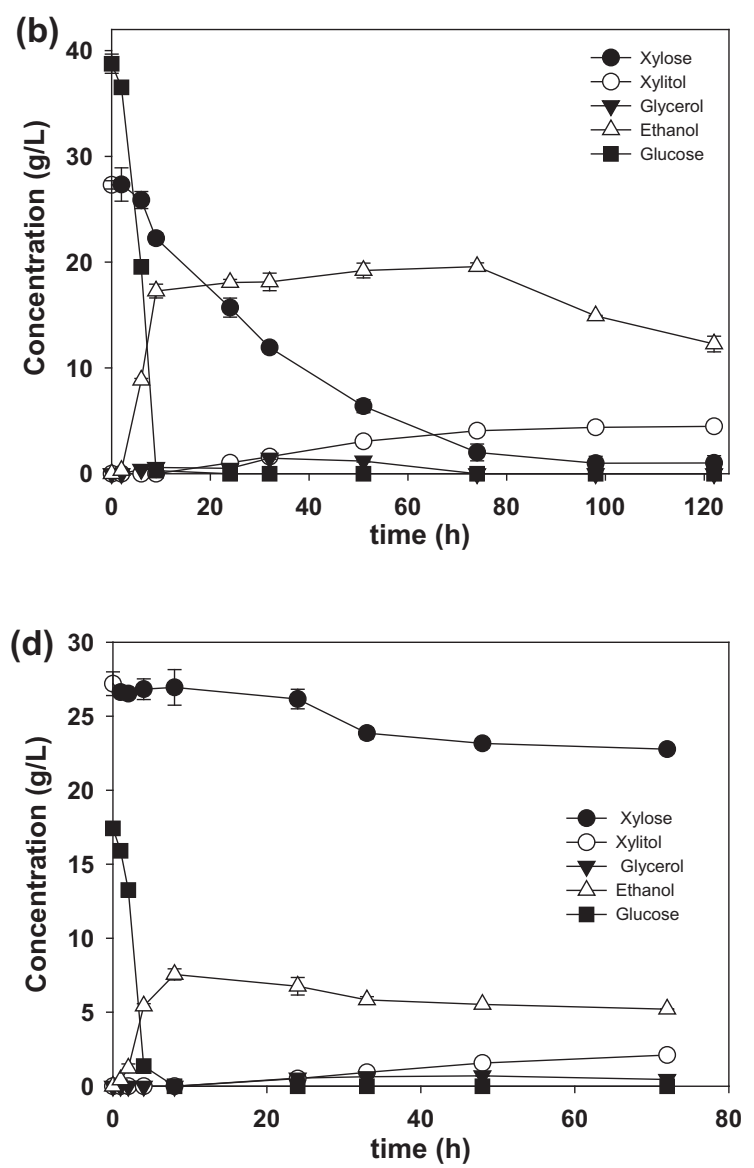

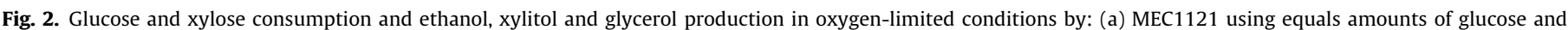

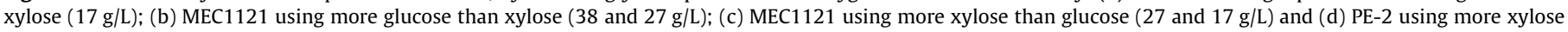
than glucose (27 and $17 \mathrm{~g} / \mathrm{L})$. 
(a)

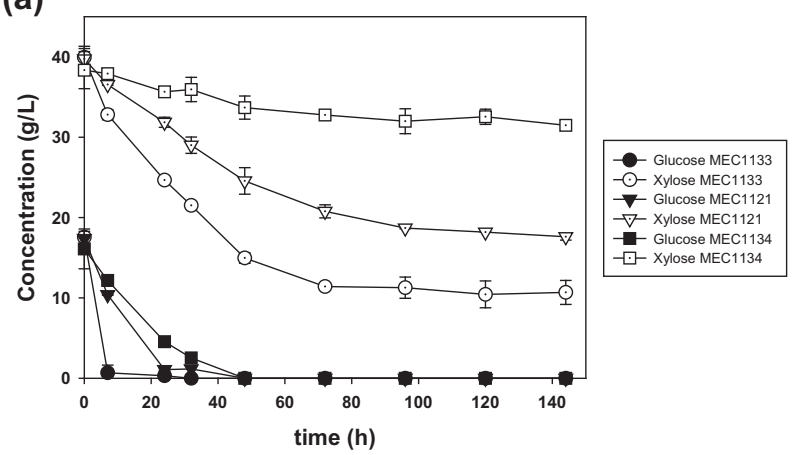

(c)

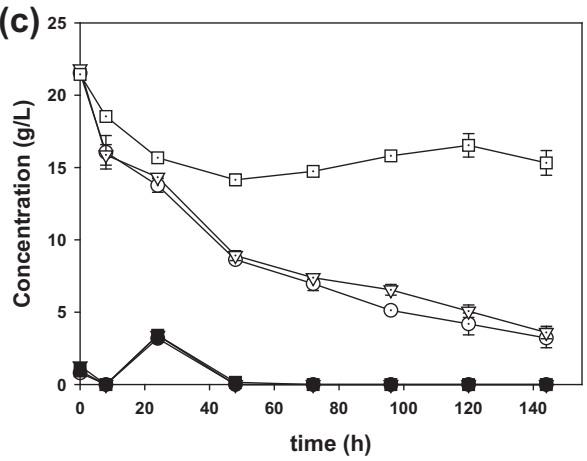

(b)

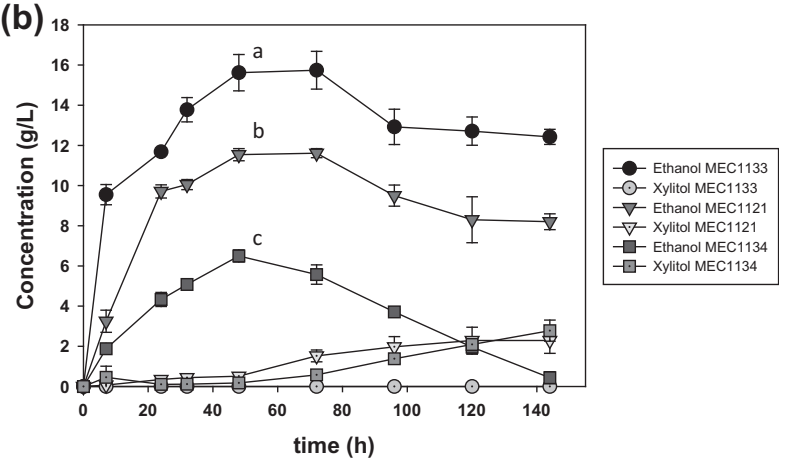

$a, b$ and $c$ indicate significant differences $(P<0.05)$ of maximun ethanol concentration produced for each strains

(d)

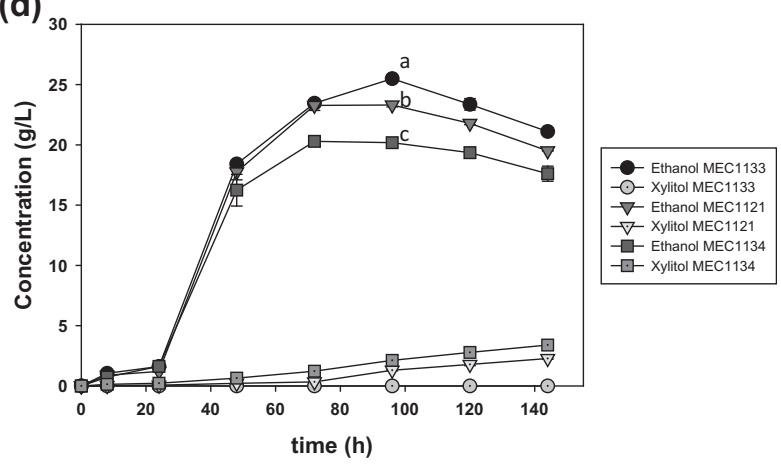

$a, b$ and $c$ indicate significant differences $(P<0.05)$ of maximun ethanol concentration produced for each strains

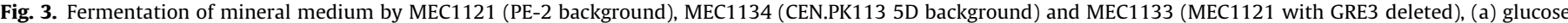

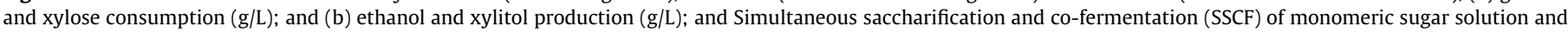
solid fraction from hydrolysis of corn cob by MEC1121, MEC1134 and MEC1133, (c) glucose and xylose consumption (g/L); and (d) ethanol and xylitol production (g/L).

corn cob autohydrolysis and monomeric sugar solution as substrate. The liquid substrate ratio (LSR) used was $15 \mathrm{~g}$ of liquid (composed by monomeric sugar solution, citrate buffer $0.05 \mathrm{~N}$, enzyme and inoculum) $/ 1 \mathrm{~g}$ of solid fraction from corn cob autohydrolysis. The enzyme employed in this work was Cellic Ctec2 (kindly provided by Novozymes, Bagsvaerd, Denmark). The cellulase activity was measured using filter paper assay (by method reported in Romaní et al., 2014). The activity of stock cellulase preparation was 120 FPU (filter paper units)/mL. Twenty FPU cellulase per gram of substrate were added to SSCF assays $24 \mathrm{~h}$ after inoculum. The samples were withdrawn periodically, centrifuged (6000 rpm for $10 \mathrm{~min}$ ) and analyzed.

\subsection{Analytical methods}

The samples from fermentation runs and SSCF assays were analyzed for xylose, glucose, xylitol, acetic acid and ethanol concentration by HPLC using a JASCO 830-IR intelligent refractive-index detector and a Metacarb $87 \mathrm{H}$ column, operating at $60{ }^{\circ} \mathrm{C}$, mobile phase $0.005 \mathrm{M} \mathrm{H}_{2} \mathrm{SO}_{4}$ and flow rate $0.7 \mathrm{~mL} / \mathrm{min}$.

\subsection{Determination of fermentation parameters}

Metabolic yield of ethanol from xylose or glucose and xylose $\left(Y_{\mathrm{E} / \mathrm{Xy}}\right.$ or $\left.Y_{\mathrm{E} / \mathrm{G}+\mathrm{Xy}}\right)$ was defined as $\mathrm{g}$ of ethanol/g of sugar consumed. Moreover, metabolic yield of xylitol from xylose $\left(Y_{\mathrm{XL} / \mathrm{Xy}}\right)$ was defined as $g$ of xylitol/g of xylose consumed. Biomass yield ( $Y_{X / S}$, $\mathrm{g} / \mathrm{g}$ ) was expressed as $\mathrm{g}$ of dry cell/g of consumed sugars. The ethanol productivity $\left(Q p_{\mathrm{t}}, \mathrm{g} / \mathrm{L} \mathrm{h}\right)$ was defined as the ethanol concentration at time $t$ divided by time $t$. Ethanol yield for simultaneous saccharification and co-fermentation $\left(Y_{\mathrm{ESSCF}}\right)$ was calculated as ethanol concentration divided by the potential glucose of solid fraction from corn cob autohydrolysis treatment and consumed xylose from monomeric sugar solution. The potential glucose (Gpot) from solid fraction of corn cob autohydrolysis was determined as follows:

$G p o t=\rho / L S R \cdot 180 / 162 \cdot G n / 100$

where $\mathrm{Gn}$ is the glucan content of solid fraction from corn cob autohydrolysis ( $\mathrm{g}$ of glucan/100 $\mathrm{g}$ of pretreated solid oven dry basis), $180 / 162$ is the stoichiometric factor, $\rho$ is the density of the saccharification and fermentation medium (average value, $1005 \mathrm{~g} / \mathrm{L}$ ), LSR is the liquid to solid ratio $(15 \mathrm{~g} / \mathrm{g})$.

\subsection{Statistical analysis}

SPSS for Windows version 21.0 was used to carry out the statistical analysis. The data are presented as means \pm standard deviation of triplicate determination. Differences among MEC1133, MEC1121 and MEC1134 strains for ethanol production in mineral medium and SSCF were tested using one-way ANOVA test, followed by Tukey's test as a post hoc comparison of means. Statistical significance was established at $P<0.05$ for the comparisons.

\section{Results and discussion}

\subsection{Strain construction}

The industrial S. cerevisiae PE-2 and CAT-1 strains were chosen for their high tolerance to inhibitory compounds (furfural, HMF 


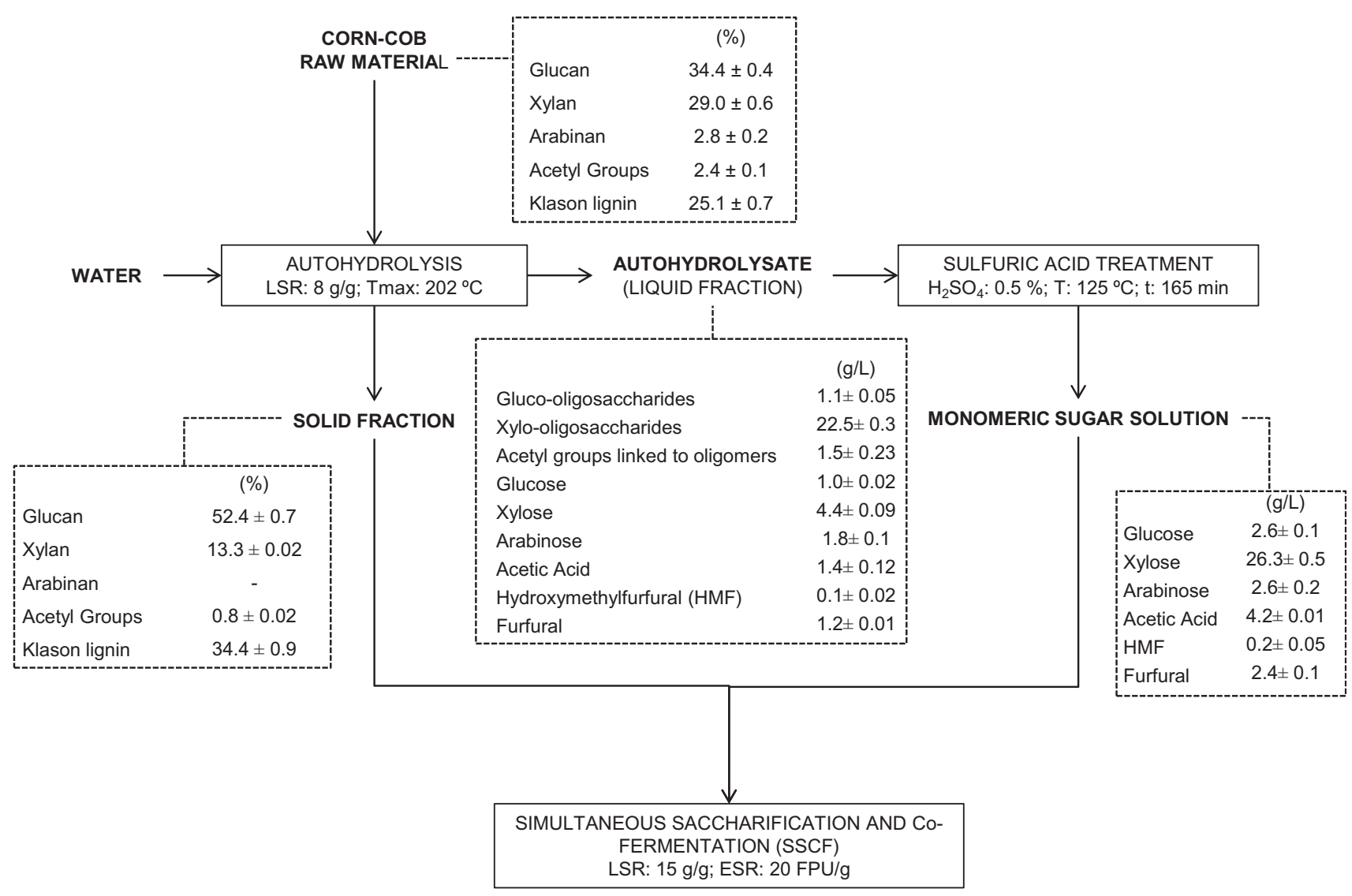

LSR: Liquid to Solid Ratio

ESR: Enzyme to Substrate Ratio

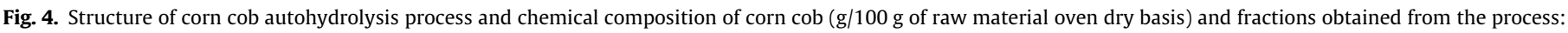
solid fraction (in $\mathrm{g} / 100 \mathrm{~g}$ of pretreated solid oven dry basis), autohydrolysate $(\mathrm{g} / \mathrm{L})$ and monomeric sugar solution ( $\mathrm{g} / \mathrm{L}$ ).

and acetic acid) and high ethanol productivities comparing to laboratory strains which was studied in previous works (Pereira et al., 2014, 2010; Romaní et al., 2014). The PE-2 and CAT-1 strains were engineered with the pMEC1049 xylose pathway which is the pMEC1035 (see Methods section for details) (Pereira et al., submitted) where the URA3 marker gene was replaced by a hygromycin resistance marker to create a dominant xylose pathway vector. The aldose reductase GRE3 was deleted from the genome in strain MEC1133. The deletions were confirmed using GRE3 flanking primers A_gre3 and D_gre3 resulting in PCR products of 1513, 1876 and $2113 \mathrm{bp}$ for the GRE3, gre3::natMX4, and gre3::kanMX4, respectively. The $S$. cerevisiae PE-2 is diploid (Argueso et al., 2009), so the two copies were deleted sequentially resulting in re3::natMX4/gre3::kanMX4 genotype. The absence of the GRE3 wild-type allele was confirmed by the disappearance of the short $1513 \mathrm{bp}$ PCR product corresponding to the wild-type locus (Fig. 1 lanes 5-8).

\subsection{Evaluation of industrial strains for xylose consumption}

Fermentation assays were carried out in anaerobic (run 1 and 2, Table 2) and oxygen-limited conditions (run 3 and 4, Table 2) for the PE-2 (MEC1121) and CAT-1 (MEC122) strain engineered with xylose pathway in order to evaluate the xylose consumption. The MEC1121 strain consumed xylose considerably faster and produced higher levels of ethanol than the MEC1122 strain. The ethanol and xylitol yields were higher for the MEC1121 than the MEC1122, as it can be seen in Table 2 (run 1-4). As expected, the fermentation rates were considerably higher and xylitol yields were lower under oxygen-limited than under anaerobic conditions for both strains (Table 2). The difference among industrial strains reveals the importance to select a host strain for developing xylose-utilizing S. cerevisiae.

\subsection{Co-fermentation of glucose and xylose mixtures}

Fermentation of both glucose and xylose simultaneously is an important point for the evaluation of production strains, since both are present in LCM hydrolysates. Since the MEC1121 showed superior xylose fermentation performance than MEC1122 strain, MEC1121 strain was chosen for further studies.

Fig. 2 shows time-course of xylose and glucose fermentation using MEC1121 (at different concentrations, Fig. 2a-c) and PE-2 (Fig. 2d) strains. In order to study the influence of different sugar concentration ratio in the fermentation of both sugars, three combinations of carbon sources were used; a balanced mixture of glucose and xylose or ratio 1:1 (Fig. 2a), higher glucose than xylose concentration or ratio $1.4: 1$ (Fig. 2b) and higher xylose than glucose concentration or ratio 1.6:1 (Fig. $2 \mathrm{c}$ and d). Surprisingly, coconsumption of glucose and xylose is observed in all conditions for the MEC1121 strain (Fig. 2a-c), most prominently during the first five hours of fermentation of the high xylose, low glucose mixture (Fig. 2c). The PE-2 strain consumed small amounts of xylose $(4.4 \mathrm{~g} / \mathrm{L})$ even without the xylose metabolic pathway (Fig. $2 \mathrm{~d}$ ). The additional xylose metabolism of the MEC1121 strain resulted in an increase of $44 \%$ in the ethanol concentration compared to the untransformed PE-2 strain after fermentation of the high xylose low glucose medium. 
Table 2

Main results of batch fermentation performance of Saccharomyces cerevisiae strains constructed in this work.

\begin{tabular}{|c|c|c|c|c|c|c|c|c|c|c|c|c|c|c|}
\hline Run & Yeast & Condition & Medium & $G_{0}(\mathrm{~g} / \mathrm{L})$ & $X y_{0}(\mathrm{~g} / \mathrm{L})$ & $G_{\mathrm{f}}(\mathrm{g} / \mathrm{L})$ & $X y_{\mathrm{f}}(\mathrm{g} / \mathrm{L})$ & $E_{48}(\mathrm{~g} / \mathrm{L})$ & $E_{\max }(\mathrm{g} / \mathrm{L})$ & $\begin{array}{l}\text { Xylitol } \\
(\mathrm{g} / \mathrm{L})\end{array}$ & $\begin{array}{l}Y_{\mathrm{E} / \mathrm{Xy}} \text { or } Y_{\mathrm{E} /} \\
\mathrm{G}+\mathrm{Xy}(\mathrm{g} / \mathrm{g})\end{array}$ & $\begin{array}{l}Y_{\mathrm{XL} / \mathrm{Xy}} \\
(\mathrm{g} / \mathrm{g})\end{array}$ & $\begin{array}{l}\mathrm{Qp}_{48} \\
(\mathrm{~g} / \\
\mathrm{L} \mathrm{h})\end{array}$ & $\begin{array}{l}Y_{\mathrm{X} / \mathrm{S}} \\
(\mathrm{g} / \mathrm{g})\end{array}$ \\
\hline 1 & MEC1121 & Anaerobic & YP & - & $45.2 \pm 0.1$ & - & $3.0 \pm 1.3$ & $5.3 \pm 0.6$ & $10.2 \pm 0.3$ & $15.2 \pm 0.7$ & 0.24 & 0.36 & 0.11 & 0.04 \\
\hline 2 & MEC1122 & Anaerobic & YP & - & $45.9 \pm 0.9$ & - & $2.4 \pm 0.2$ & $2.5 \pm 0.3$ & $11.1 \pm 0.2$ & $12.7 \pm 0.1$ & 0.25 & 0.29 & 0.05 & 0.04 \\
\hline 3 & MEC1121 & $\begin{array}{l}\text { Oxygen- } \\
\text { limited }\end{array}$ & YP & - & $44.0 \pm 0.0$ & - & $0.4 \pm 0.0$ & $8.1 \pm 0.2$ & $11.0 \pm 0.4$ & $0.5 \pm 0.5$ & 0.25 & 0.01 & 0.17 & 0.20 \\
\hline 4 & MEC1122 & $\begin{array}{l}\text { Oxygen- } \\
\text { limited }\end{array}$ & YP & - & $43.7 \pm 0.6$ & - & $2.1 \pm 0.3$ & $7.9 \pm 0.7$ & $9.7 \pm 0.7$ & $2.9 \pm 0.2$ & 0.23 & 0.07 & 0.16 & 0.25 \\
\hline 5 & MEC1121 & $\begin{array}{l}\text { Oxygen- } \\
\text { limited }\end{array}$ & YP & $17.6 \pm 1.3$ & $16.8 \pm 0.4$ & $0.0 \pm 0.0$ & $0.4 \pm 0.8$ & $10.9 \pm 0.9$ & $11.7 \pm 0.1$ & $1.9 \pm 0.1$ & 0.34 & 0.11 & 0.23 & 0.18 \\
\hline 6 & MEC1121 & $\begin{array}{l}\text { Oxygen- } \\
\text { limited }\end{array}$ & YP & $38.8 \pm 0.9$ & $27.3 \pm 0.4$ & $0.0 \pm 0.0$ & $2.0 \pm 0.8$ & $19.2 \pm 0.7$ & $19.6 \pm 0.4$ & $4.5 \pm 0.2$ & 0.31 & 0.18 & 0.40 & 0.19 \\
\hline 7 & MEC1121 & $\begin{array}{l}\text { Oxygen- } \\
\text { limited }\end{array}$ & YP & $17.3 \pm 0.0$ & $26.9 \pm 0.4$ & $0.0 \pm 0.0$ & $7.4 \pm 0.9$ & $10.9 \pm 0.8$ & $10.9 \pm 0.8$ & $2.8 \pm 0.0$ & 0.30 & 0.14 & 0.23 & 0.23 \\
\hline 8 & MEC1121 & $\begin{array}{l}\text { Oxygen- } \\
\text { limited }\end{array}$ & $\mathrm{SH}$ & $1.71 \pm 0.0$ & $29.9 \pm 0.6$ & $0.0 \pm 0.0$ & $3.43 \pm 1.7$ & $8.1 \pm 0.7$ & $8.1 \pm 0.3$ & $4.6 \pm 0.4$ & 0.29 & 0.17 & 0.17 & 0.15 \\
\hline 9 & MEC1133 & $\begin{array}{l}\text { Oxygen- } \\
\text { limited }\end{array}$ & MM & $17.6 \pm 0.1$ & $39.9 \pm 1.4$ & $0.0 \pm 0.0$ & $11.4 \pm 1.5$ & $15.6 \pm 0.9$ & $15.7 \pm 0.9$ & $0.0 \pm 0.0$ & 0.34 & 0.00 & 0.33 & 0.15 \\
\hline 10 & MEC1121 & $\begin{array}{l}\text { Oxygen- } \\
\text { limited }\end{array}$ & MM & $17.4 \pm 0.9$ & $39.7 \pm 1.3$ & $0.0 \pm 0.0$ & $20.8 \pm 0.4$ & $11.5 \pm 0.3$ & $11.6 \pm 0.23$ & $1.5 \pm 0.6$ & 0.32 & 0.08 & 0.24 & 0.19 \\
\hline 11 & MEC1134 & $\begin{array}{l}\text { Oxygen- } \\
\text { limited }\end{array}$ & MM & $16.1 \pm 2.5$ & $38.3 \pm 0.5$ & $0.0 \pm 0.0$ & $32.8 \pm 2.3$ & $6.5 \pm 0.3$ & $5.6 \pm 0.5$ & $0.6 \pm 0.5$ & 0.26 & 0.10 & 0.14 & 0.13 \\
\hline
\end{tabular}

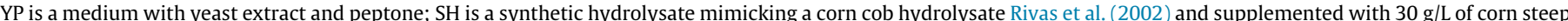

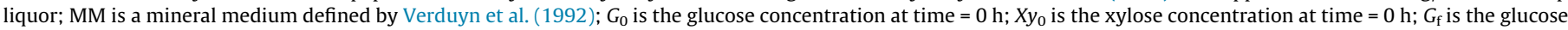

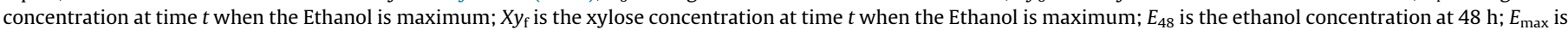

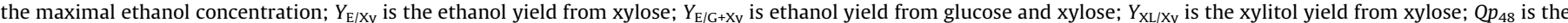
productivity of ethanol at $48 \mathrm{~h} ; Y_{\mathrm{X} / \mathrm{S}}$ is the biomass yield at end of fermentation ( $\mathrm{g}$ of dry cell/g of consumed sugar).

In recent works, laboratory strains of $S$. cerevisiae have been engineered for co-fermentation of glucose and xylose (Gonçalves et al., 2014; Zha et al., 2014). Gonçalves et al. (2014) transformed a hxt-null strain lacking the major hexose transporters with an integrated plasmid to overexpress the XYL1, XYL2 and XKS1genes. The results obtained for co-fermentation of equal glucose and xylose concentrations showed ethanol yield of $0.39 \mathrm{~g} / \mathrm{g}$ with a glucose and xylose consumption of $92 \%$ and $46 \%$, respectively, using a MA-B43 strain. These results can be compared with the ones obtained in this work (see Table 1, run 5) in which an ethanol yield of $0.34 \mathrm{~g} / \mathrm{g}$ with a glucose and xylose consumption of $100 \%$ and $98 \%$ respectively was achieved. On the other hand, an ethanol yield of $0.37 \mathrm{~g} / \mathrm{g}$ was reported by Zha et al. (2014) with a xylose consumption of $97 \%$ using a mutant strain SyBE005 (mutant obtained from an evolutionary engineering experiment on xylose with the genetically engineered strain SyBE004).

\subsection{Fermentation of a synthetic hydrolysate}

A synthetic hydrolysate was prepared mimicking a corn cob hydrolysate (Rivas et al., 2002) in order to test performance in a situation more closely reflecting an industrial fermentation. Xylose consumption rate can be particularly reduced during fermentation of lignocellulose hydrolysates (Jin et al., 2012). The fermentation of synthetic hydrolysate by MEC1121 showed very little xylose consumption, possibly due to the absence of a nitrogen source or other nutrients (Fig. S1, Supplementary materials). Yeast extract and peptone are not normally used industrially due to high cost. Corn steep liquor (CSL) is a by-product of corn starch processing and is a low cost source of proteins, amino acids and other nutrients. CSL has been used as an effective replacement for yeast extract and peptone in alcoholic fermentation (Pereira et al., 2010). An additional fermentation of synthetic hydrolysate supplemented with CSL was performed. The main results obtained are listed in Table 2 (see run 8). The MEC1121 was able to efficiently ferment xylose in the presence of inhibitory compounds $(2.3 \mathrm{~g} / \mathrm{L}$ of acetic acid, $1.2 \mathrm{~g} / \mathrm{L}$ of furfural and $0.1 \mathrm{~g} / \mathrm{L}$ HMF) with ethanol yields and productivities very similar to results obtained with pure xylose supplemented with yeast extract and peptone (run 3 ), as it can be observed in Table 2. The Qp at $48 \mathrm{~h}$ was $0.16 \mathrm{~g} / \mathrm{L} \mathrm{h}$ using synthetic hydrolysate supplemented with CSL (Table 2). The PE-2 based strains MEC1121 performed well in the presence of inhibitors as expected since this strain background was found to be among the most resistant in a selection of ten industrial and four laboratory strains (Pereira et al., 2014). The PE-2 was shown to be able to reduce furfural and HMF content very quickly and faster than an industrial yeast strain isolated from a 2nd generation bioethanol plant (Pereira et al., 2014).

\subsection{Comparison of strain backgrounds for xylose fermentation}

The laboratory strain MEC1134 (CEN.PK113 5D carrying the same xylose pathway as MEC1121) was used in a direct comparison with MEC1121 to obtain a measure of the relative efficiency of the PE-2 background for xylose fermentation. A commonly used mineral medium (Verduyn et al., 1992) containing $17 \mathrm{~g} / \mathrm{L}$ glucose and $40 \mathrm{~g} / \mathrm{L}$ xylose was used to facilitate comparison with previously described strains (Träff et al., 2001). The MEC1121 consumed significantly more xylose than the MEC1134, indicating that the PE-2 strain background has an intrinsic ability to efficiently ferment xylose. Glucose was consumed within $30-40 \mathrm{~h}$ for both strains. The MEC1121 strain produced almost twice the amount of ethanol compared to the MEC1134 strain (Fig. 3b). The high production of xylitol obtained by MEC1121 strain compared to the MEC1122 (Table 2) suggested that the MEC1121 could be engineered for lower xylitol yield. The MEC1133 is the MEC1121 with both alleles of the GRE3 gene deleted. The deletion of GRE3 resulted in faster xylose consumption (comparing strains MEC1121 and MEC1133 in Fig. 3a). Ethanol production was also improved and xylitol yield were lowered to undetectable levels (comparing the same strains in Fig. 3b). Ethanol concentrations produced by MEC1133, MEC1121 and MEC1134 strains were analyzed statistically and significant differences $(P<0.05)$ were observed as can be showed in Fig. 3b. Final ethanol concentrations (Fig. 3b) 
obtained by MEC1133 and MEC1121 strains were 15.74 and $11.61 \mathrm{~g} / \mathrm{L}$, corresponding to ethanol productivities of 0.33 and $0.24 \mathrm{~g} /(\mathrm{L} \mathrm{h})$, respectively (see Table 2). This shows that the GRE3 deletion led to higher productivity. The ethanol yields were comparable $\left(Y_{E / G+X}=0.34\right.$ for the MEC1133 and 0.32 for the MEC1121), as both xylose consumption and ethanol production improved.

The S. cerevisiae PE-2 based xylose fermenting strains MEC1121 and MEC1133 constructed as a part of this work displayed a striking ability to efficiently consume xylose without adaptation. In a recent work, Zha et al. (2014) have obtained an evolved strain with a 2.6-fold higher specific ethanol production rate in xylose when compared to the non-adapted recombinant strain. These authors have identified point mutations in the transcriptional regulators CYC8 and PHD, and in ZWF1 and suggested that these might be the cause of the upregulation of genes involved in xylose metabolism. Thus, the PE-2 strain genetic background might have specific attributes that renders the recombinant strain efficient in fermenting xylose without any adaptation. On the other hand, the recombinant PE-2 based strains also showed the ability to co-consume xylose and glucose. The CEN.PK based laboratory strain MEC1134 was unable to consume xylose in the presence of glucose under the same conditions. The normal observation is that xylose consumption stalls until glucose levels reach a low level, possibly due to competition for transporter capacity as glucose and xylose are transported by the same membrane proteins (Gonçalves et al., 2014; Kim et al., 2013). Non-recombinant strategies have been recently reported in order to overcome this limitation of up-to-now engineered strains, but require the encapsulation of the $S$. cerevisiae cells (Westman et al., 2014).

The comparison between MEC1121 and MEC1134 represents a direct comparison between the two strain backgrounds, as the pathway vector is identical and the strains were only subjected to one transformation step. Thus, the ability of co-fermenting glucose and xylose is intrinsic to the PE-2 background. A number of genes are differentially expressed comparing the genome reference strain S288c and the PE-2, and sixteen additional genes are present in the PE-2 genome of which at least two are transporters and six are uncharacterized (Argueso et al., 2009). Xylose uptake has been identified as limiting xylose fermentation rate particularly in the presence of glucose independently of the xylose metabolic pathway (Fonseca et al., 2011). Thus, an altered transport capacity for xylose and glucose might contribute to this particularity of the PE-2 based strain.

Moreover, the MEC1121 (PE-2 background) consumed xylose faster than the MEC1122 (CAT1 background), but also produced more xylitol. Our results show that rapid xylose consumption rate might be a more important trait for strain selection than low xylitol production as xylitol formation can be eliminated by GRE3 deletion. The GRE3 deletion also had the additional benefit of a slightly faster xylose consumption rate. The MEC1133 strain represents the first description of an industrial xylose fermenting $S$. cerevisiae strain with a complete deletion of the GRE3/YHR104w aldose reductase. The industrial strain CIBTS0573 (Diao et al., 2013) was engineered for xylose fermentation, and one of the GRE3 alleles was disrupted in the process. However, this strain did not consume xylose without adaptation.

\subsection{Preparation and characterization of a corn-cob hydrolysate}

Corn-cob is a raw material that is easily incorporated in the industrial production of ethanol from corn, provided that fermentation strains able to cope with this substrate are available. Corn cob can be hydrolyzed in a two step process, where the initial hydrolysis is performed in the presence of water only (Romaní et al., 2014) in a process called autohydrolysis. The liquid fraction following the autohydrolysis (denoted autohydrolysate) is rich in xylan derived from hemicellulose which solubilize more easily than cellulose. Corn cob autohydrolysis were performed as previously described (Romaní et al., 2014). The hydrolysis strategy and the chemical composition of each fraction are described in Fig. 4. Seventy percent of the xylan fraction was solubilized in the autohydrolysate as monosaccharides and oligosaccharides. The solid fraction was enriched in glucan and lignin. The recovery of glucan and lignin were $98 \%$ and $89 \%$, respectively (see Supplementary material for calculations). Oligosaccharides in the autohydrolysate were completely digested into monomers by sulfuric acid treatment. Acid treatment increased acetic acid concentration, a consequence of hydrolysis of acetyl groups linked to xylooligosaccharides. Furfural and hydroxymethylfurfural content was also higher due to the dehydration of xylose and glucose.

\subsection{Simultaneous saccharification and co-fermentation of corn cob}

The solid fraction and the monomeric sugar solution (Fig. 4) were combined into a substrate for simultaneous saccharification and co-fermentation of glucose and xylose (SSCF). The SSCF experiments were carried with $15 \mathrm{~g}$ of liquid (monomeric sugar solution, buffer citrate, enzyme and inoculum) per gram of solid fraction. Cellulase was added $24 \mathrm{~h}$ after the initiation.

The time course of concentrations of substrates and products of SSCF processes are shown in Fig. $3 c$ and d. Glucose concentration was only measurable right after cellulase addition (Fig. 3c), but otherwise undetectable indicating that glucose was consumed as it was liberated. The xylose consumption profiles of MEC1133 and MEC1121 were very similar with a consumed xylose percentage of $58 \%$ and $59 \%$ at $48 \mathrm{~h}$, respectively. The laboratory strain MEC1134 consumed xylose only in the first $24 \mathrm{~h}$ (before the addition of cellulase). Xylitol production for all strains were below five grams per liter, MEC1133 producing levels below detection limits similarly to the fermentation of xylose glucose mixtures (Fig. 3d). The MEC1133 produced the highest concentration of ethanol $(25.5 \mathrm{~g} / \mathrm{L})$ of the three strains. The maximum ethanol concentration of three strains was compared and significant differences were observed $(P<0.05)$, see Fig. $3 \mathrm{~d}$. This represents an improvement of $25 \%$ over the MEC1134 laboratory strain. The ethanol yield ( $Y_{\mathrm{ESS}-}$ CF) was higher for the MEC1133 at $0.47 \mathrm{~g} / \mathrm{g}$ compared to 0.45 for the MEC1121 indicating that there is also a beneficiary effect of this deletion for SSCF. The ethanol yields with SSCF were higher than yields obtained with batch fermentations. This could be due to the simultaneous liberation and consumption of glucose leading to very low free glucose concentration. This was also observed before in SSCF of substrate wheat straw (Olofsson et al., 2010).

Similar works are collected in the literature. SSCF of steam exploded wheat straw resulted in an ethanol yield of $0.33 \mathrm{~g} / \mathrm{g}$ and fermentation of $43-46 \%$ of available xylose by an adapted engineered S. cerevisiae strain (Tomás-Pejó et al., 2014). For corn cobs raw material, an ethanol yield of $0.28 \mathrm{~g} / \mathrm{g}$ was recently reported using the $S$. cerevisiae KE6-12 strain, an evolved strain derived from TMB 3400 (Wahlbom et al., 2003). In the present study, SSCF of corn-cob hydrolysate resulted in an ethanol yield of $0.47 \mathrm{~g} / \mathrm{g}$ of total sugars using MEC1133 which represents $92 \%$ of the theoretical yield. A direct comparison across results found in literature is not straightforward as different raw materials, pretreatments, solid loads, enzymes, and processes have been used. Nevertheless, the yield presented here is unquestionable high when comparing to yields obtained with SSCF found in literature (Tomás-Pejó et al., 2014; Novy et al., 2013; van Eylen et al., 2011), this without any specific process optimization. This result highlights that industrial strain backgrounds represent a yet largely unexplored resource for the continued development of processes for second generation bioethanol production. 


\section{Conclusions}

Xylose fermentation could improve overall process economy of the second generation bioethanol. We have engineered xylose metabolic pathway in industrial robust strains previously studied for tolerance to inhibitors. Industrial strains efficiently consumed xylose in presence of inhibitors in different media. Unexpectedly, while glucose was consumed faster than xylose, we observed xylose and glucose co-fermentation regardless of initial ratio between the sugars. This shows that yet uncharacterized S. cerevisiae strain backgrounds may have a wider metabolic range than previously thought and that the continued characterization of wild-type or adapted strains may be important for the future development of efficient yeast based processes.

\section{Acknowledgements}

This work was supported by the Fundação para a Ciência $e$ Tecnologia (FCT) through projects RECI/BBB-EBI/0179/2012FCOMP-01-0124-FEDER-027462, PEst-OE/EQB/LA0023/2013, "BioInd - Biotechnology and Bioengineering for improved Industrial and Agro-Food processes, REF. NORTE-07-0124-FEDER-000028" Co-funded by the Programa Operacional Regional do Norte (ON.2 - O Novo Norte) QREN, FEDER, PTDC/AAC-AMB/120940/2010, EXPL/BBB-BIO/1772/2013 and the FEDER POFC-COMPETE (PEst-C/ BIA/UI4050/2011). A.R. was supported by FCT fellowship [SFRH/ BPD/77995/2011] and F.P. by FCT fellowships [SFRH/BD/80934/ 2011 and SFRH/BD/42565/2007].

\section{Appendix A. Supplementary data}

Supplementary data associated with this article can be found, in the online version, at http://dx.doi.org/10.1016/j.biortech.2014. 12.020 .

\section{References}

Argueso, J.L., Carazzolle, M.F., Mieczkowski, P.A., Duarte, F.M., Netto, O.V.C., Missawa, S.K., Galzerani, F., Costa, G.G.L., Vidal, R.O., Noronha, M.F., Dominska, M., Andrietta, M.G.S., Andrietta, S.R., Cunha, A.F., Gomes, L.H., Tavares, F.C.A. Alcarde, A.R., Dietrich, F.S., McCusker, J.H., Petes, T.D., Pereira, G.A.G., 2009. Genome structure of a Saccharomyces cerevisiae strain widely used in bioethanol production. Genome Res. 19, 2258-2270.

Basso, L.C., de Amorim, H.V., de Oliveira, A.J., Lopes, M.L., 2008. Yeast selection for fuel ethanol production in Brazil. FEMS Yeast Res. 8, 1155-1163.

Diao, L., Liu, Y., Qian, F., Yang, J., Jiang, Y., Yang, S., 2013. Construction of fast xylosefermenting yeast based on industrial ethanol-producing diploid Saccharomyces cerevisiae by rational design and adaptive evolution. BMC Biotechnol. 13, 110.

Fonseca, C., Olofsson, K., Ferreira, C., Runquist, D., Fonseca, L.L., Hahn-Hägerdal, B., Lidén, G., 2011. The glucose/xylose facilitator Gxf1 from Candida intermedia expressed in a xylose-fermenting industrial strain of Saccharomyces cerevisiae increases xylose uptake in SSCF of wheat straw. Enzyme Microb. Technol. 48, 518-525.

Gietz, R.D., Schiestl, R.H., 2007. High-efficiency yeast transformation using the LiAc/ SS carrier DNA/PEG method. Nat. Protoc. 2, 31-34.

Goldstein, A.L., McCusker, J.H., 1999. Three new dominant drug resistance cassettes for gene disruption in Saccharomyces cerevisiae. Yeast 15, 1541-1553.

Gonçalves, D.L., Matsushika, A., de Sales, B.B., Goshima, T., Bon, E.P.S., Stambuk, B.U., 2014. Xylose and xylose/glucose co-fermentation by recombinant Saccharomyces cerevisiae strains expressing individual hexose transporters. Enzyme Microb. Technol. 63, 13-20.

Hahn-Hägerdal, B., Pamment, N., 2004. Special session A microbial pentose metabolism. Appl. Biochem. Biotechnol. 116, 1207-1209.

Hinman, N.D., Wright, J.D., Hogland, W., Wyman, C.E., 1989. Xylose fermentation: an economic analysis. Appl. Biochem. Biotechnol. 20-21, 391-401.

Hoffman, C.S., 2001. Preparation of yeast DNA. In: Ausubel, F.M., Brent, R., Kingston, R.E., Moore, D.D., Seidman, J.G., Smith, J.A., Struhl, K. (Eds.), Current Protocols in Molecular Biology. John Wiley \& Sons, Inc.; Unit 13.11.

Inoue, H., Nojima, H., Okayama, H., 1990. High efficiency transformation of Escherichia coli with plasmids. Gene 96, 23-28.

Jin, M., Balan, V., Gunawan, C., Dale, B.E., 2012. Quantitatively understanding reduced xylose fermentation performance in AFEXTM treated corn stover hydrolysate using Saccharomyces cerevisiae 424A (LNH-ST) and Escherichia coli K011. Bioresour. Technol. 111, 294-300.

Kim, S.R., Park, Y.-C., Jin, Y.-S., Seo, J.-H., 2013. Strain engineering of Saccharomyces cerevisiae for enhanced xylose metabolism. Biotechnol. Adv. 31, 851-861.

Kötter, P., Amore, R., Hollenberg, C.P., Ciriacy, M., 1990. Isolation and characterization of the Pichia stipitis xylitol dehydrogenase gene, XYL2, and construction of a xylose-utilizing Saccharomyces cerevisiae transformant. Curr. Genet. 18, 493-500.

Kuyper, M., Harhangi, H.R., Stave, A.K., Winkler, A.A., Jetten, M.S.M., de Laat, W.T.A.M., den Ridder, J.J.J., Op den Camp, H.J.M., van Dijken, J.P., Pronk, J.T., 2003. High-level functional expression of a fungal xylose isomerase: the key to efficient ethanolic fermentation of xylose by Saccharomyces cerevisiae? FEMS Yeast Res. 4, 69-78.

Lindén, T., Peetre, J., Hahn-Hägerdal, B., 1992. Isolation and characterization of acetic acid-tolerant galactose-fermenting strains of Saccharomyces cerevisiae from a spent sulfite liquor fermentation plant. Appl. Environ. Microbiol. 58, $1661-1669$.

Novy, V., Krahulec, S., Longus, K., Klimacek, M., Nidetzky, B., 2013. Co-fermentation of hexose and pentose sugars in a spent sulfite liquor matrix with genetically modified Saccharomyces cerevisiae. Bioresour. Technol. 130, 439-448.

Olofsson, K., Palmqvist, B., Lidén, G., 2010. Improving simultaneous saccharification and co-fermentation of pretreated wheat straw using both enzyme and substrate feeding. Biotechnol. Biofuels 3, 17.

Pereira, F., Parachin, N.S., Hahn-Hägerdal, B., Gorwa-Grauslund, M.F., Johansson, B. Unpublished results. The Yeast Pathway Kit: a method for rational or combinatorial metabolic pathways design in Saccharomyces cerevisiae with software support, Submitted.

Pereira, F.B., Guimarães, P.M.R., Teixeira, J.A., Domingues, L., 2010. Optimization of low-cost medium for very high gravity ethanol fermentations by Saccharomyces cerevisiae using statistical experimental designs. Bioresour. Technol. 101, 78567863.

Pereira, F.B., Guimarães, P.M.R., Teixeira, J.A., Domingues, L., 2011. Robust industrial Saccharomyces cerevisiae strains for very high gravity bio-ethanol fermentations. J. Biosci. Bioeng. 112, 130-136.

Pereira, F.B., Romaní, A., Ruiz, H.A., Teixeira, J.A., Domingues, L., 2014. Industria robust yeast isolates with great potential for fermentation of lignocellulosic biomass. Bioresour. Technol. 161, 192-199.

Rivas, B., Dominguez, J.M., Domínguez, H., Parajó, J.C., 2002. Bioconversion of posthydrolysed autohydrolysis liquors: an alternative for xylitol production from corn cobs. Enzyme Microb. Technol. 31, 431-438.

Romaní, A., Ruiz, H.A., Pereira, F.B., Teixeira, J.A., Domingues, L., 2014. Integrated approach for effective bioethanol production using whole slurry from autohydrolyzed Eucalyptus globulus wood at high-solid loadings. Fuel 135, 482-491.

Runquist, D., Hahn-Hägerdal, B., Bettiga, M., 2010. Increased ethanol productivity in xylose-utilizing Sacharomyces cerevisiae via randomly mutagenized xylose reductase. Appl. Environ. Microbiol. 76, 7796-7802.

Sonderegger, M., Jeppsson, M., Larsson, C., Gorwa-Grauslund, M.-F., Boles, E., Olsson, L., Spencer-Martins, I., Hahn-Hägerdal, B., Sauer, U., 2004. Fermentation performance of engineered and evolved xylose-fermenting Saccharomyces cerevisiae strains. Biotechnol. Bioeng. 87, 90-98.

Tomás-Pejó, E., Bonander, N., Olsson, L., 2014. Industrial yeasts strains for biorefinery solutions: constructing and selecting efficient barcoded xylose fermenting strains for ethanol. Biofuels Bioprod. Biorefin. 8, 626-634.

Träff, K.L., Otero Cordero, R.R., van Zyl, W.H., Hahn-Hägerdal, B., 2001. Deletion of the GRE3 aldose reductase gene and its influence on xylose metabolism in recombinant strains of Saccharomyces cerevisiae expressing the xylA and XKS1 genes. Appl. Environ. Microbiol. 67, 5668-5674.

Van der Westhuizen, T.J., Pretorius, I.S., 1992. The value of electrophoretic fingerprinting and karyotyping in wine yeast breeding programmes. Antonie Van Leeuwenhoek 61, 249-257.

van Dijken, J.P., Bauer, B., Duboc, F., Gancedo, G., Heijnen, H., Lange, M. Niederberger, N., Parrou, P., Porro, R., van Riel N, R., Steensma, V., Vindeløv, P., 2000. An interlaboratory comparison of physiological and genetic properties of four Saccharomyces cerevisiae strains. Enzyme Microb. Technol. 26, 706-714.

van Eylen, D., van Dongen, F., Kabel, M., de Bont, J., 2011. Corn fiber, cobs and stover: enzyme-aided saccharification and co-fermentation after dilute acid pretreatment. Bioresour. Technol. 102, 5995-6004.

Verduyn, C., Postma, E., Scheffers, W.A., Van Dijken, J.P., 1992. Effect of benzoic acid on metabolic fluxes in yeasts: a continuous-culture study on the regulation of respiration and alcoholic fermentation. Yeast 8, 501-517.

Wahlbom, C.F., van Zyl, W.H., Jönsson, L.J., Hahn-Hägerdal, B, Otero, R.R.C., 2003. Generation of the improved recombinant xylose-utilizing Saccharomyces cerevisiae TMB 3400 by random mutagenesis and physiological comparison with Pichia stipitis CBS 6054. FEMS Yeast Res. 3, 319-326.

Westman, J., Bonander, N., Taherzadeh, M., Franzen, C.J., 2014. Improved sugar coutilisation by encapsulation of a recombinant Saccharomyces cerevisiae strain in alginate-chitosan capsules. Biotechnol. Biofuels 7, 102.

Zha, J., Shen, M., Hu, M., Song, H., Yuan, Y., 2014. Enhanced expression of genes involved in initial xylose metabolism and the oxidative pentose phosphate pathway in the improved xylose-utilizing Saccharomyces cerevisiae through evolutionary engineering. J. Ind. Microb. Biotechnol. 41, 27-39. 